\title{
Inferior Vena Cava Thrombosis Following a Traumatic Liver Injury
}

\author{
Pil Young Jung \\ Department of Surgery, Yonsei university Wonju college of medicine, Wonju Severance Christian Hospital, Trauma center, Wonju, Korea \\ Thrombosis within the inferior vena cava (IVC) following liver injury is an extremely rare condition. However, while the \\ incidence of severe post-traumatic venous thrombosis is not high, it can cause fatal complications such as pulmonary \\ embolism. Thus, it remains a therapeutic challenge in a traumatic context because of the hemorrhagic risk due to \\ anticoagulation. Here we report a case of thrombosis from the iliac vein to the inferior vena cava following hepatic injury. \\ (Trauma Image Proced 2017(2):62-63)
}

Key Words: Inferior vena cava thrombosis; Liver injury

\section{CASE}

A 25-year-old man visited the hospital due to abdominal trauma caused by a traffic accident. His vital signs were normal, and he complained of pains in the abdomen, neck and right foot. Abdominal computed tomography showed a hepatic injury and a conservative treatment was performed in the intensive care unit. One week later, a follow up abdominal computed tomography revealed thrombosis from the iliac vein to the inferior vena cava which prompted heparin therapy. Two weeks after admission, the patient underwent an operation on a right metatarsal fracture, and a follow up abdominal computed tomography scan was performed to observe thrombosis. Thrombosis was found to be elevated, and an IVC filter was inserted into the inferior vena cava. Concomitant use of heparin, followed by conservative treatment with coumadin, continued the 28th day following abdominal computed tomography, which resulted in abatement of the thrombosis. The patient was discharged on the 34th day after admission without further complications.

\section{DISCUSSION}

Although the incidence of severe post-traumatic venous thrombosis is not high, it may lead to fatal complications such as pulmonary embolism $(1,2)$. Therefore, early detection and appropriate treatment are required. Prophylactic treatment is warranted to identify risk factors that increase the likelihood of thrombosis, and active treatment is required when this occurs.

Conflict of Interest Statement

None of authors has a conflict of interest.

Received: November 1, 2017 Revised: November 13, 2017 Accepted: November 13, 2017

Correspondence to: Pil Young Jung, Department of Surgery, Yonsei University Wonju College of Medicine, Wonju Severance Christian Hospital, 20 Ilsan-ro, Wonju-si, Gangwon-do, Republic of Korea

Tel: 82-33-741-0882, Fax: 82-33-741-1205, E-mail: surgery4trauma@yonsei.ac.kr

Copyright (c) 2017 Korean Association for Research, Procedures and Education on Trauma. All rights reserved.

@This is an open-access article distributed under the terms of the Creative Commons Attribution Non-Commercial License (http://creativecommons.org/ licenses/by-nc/4.0) which permits unrestricted noncommercial use, distribution, and reproduction in any medium, provided the original work is properly cited 


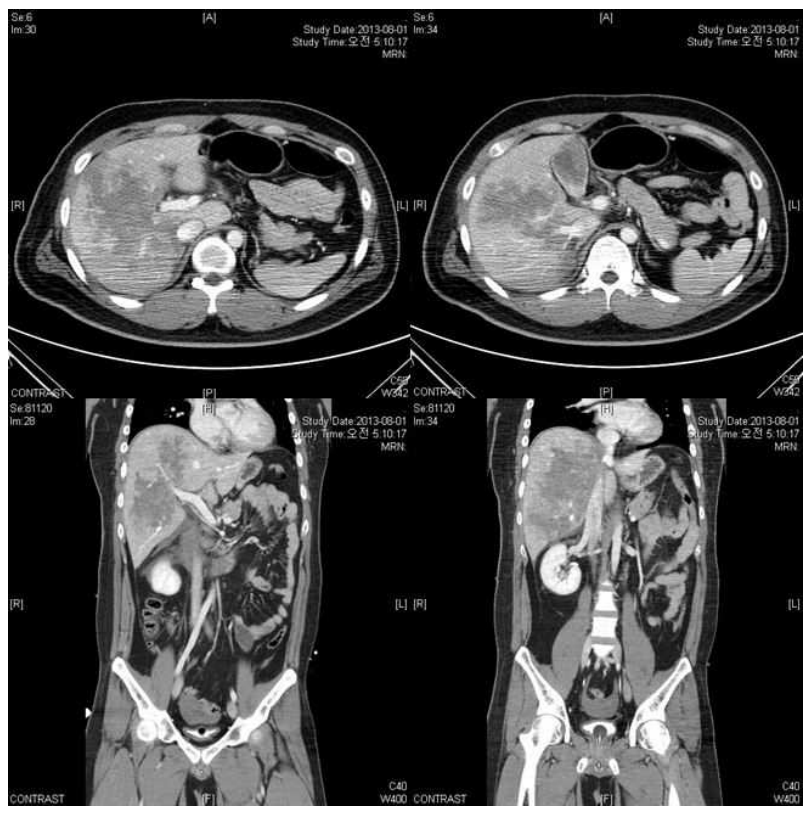

Fig. 1. CT scan of the abdomen-pelvis on admission day shows traumatic liver injury.



Fig. 3. IVC filter insertion

\section{REFERENCES}

1. Chakroun A, Nakhli MS, Kahloul M, Harrathi MA, Naija W. Post traumatic inferior vena cava thrombosis: A case report and review of literature. Int J Surg Case Rep. 2017;36:59-63.

2. Kim KY, So BJ, Park DE. Management of inferior vena cava thrombosis after blunt liver injury. Korean J Hepatobiliary Pancreat Surg. 2014 Aug;18(3):97-100.

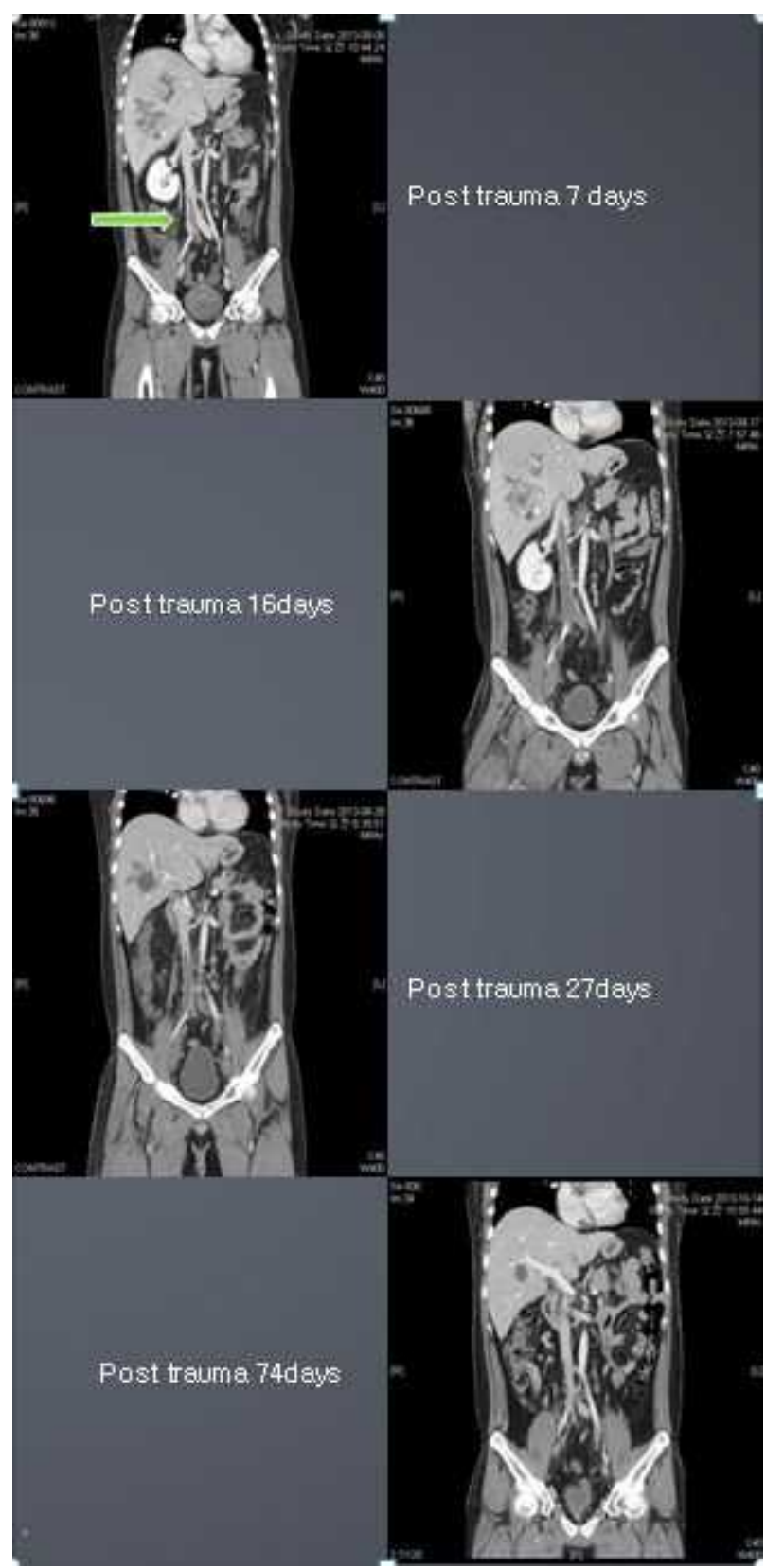

Fig. 2. Serial CT scan shows the change of the thrombosis. 